\title{
Subject Index-Volume 2, 2001
}

A3243G mitochondrial DNA

mutation, 172

Acoustic reflex, 31

Across-channel temporal judgments, 388

Across-frequency, 388

Age, 388

Age-related hearing loss, 118, 233

Apoptosis, 79

ATP, 362, 399

Auditory brainstem response, 130

Auditory cortex, 199, 279, 297

Auditory filters, 408

Auditory function, 130

Auditory nerve, 41

Auditory nerve fiber, 216

Auditory neurons, 180

Auditory profile analysis, 189

Auditory system function, 312, 330, 348

Avian auditory inner ear epithelia, 79

Avian cochlear nucleus complex, 41

Axonal neurofilament expression, 297

Azimuth localization, 246

BALB/cByJ mice, 233

Barn owl, 1, 41

Basilar membrane, 257

Belgian Waterslager canary, 79

Bicuculline, 279

Bone remodeling, 65

Bone resorption, 65

Broadband noise, 246

C57BL/ 6 mice, 118

C57BL/6J mice, 233

Calcium homeostasis, 180

Caloric test, 72

Canal paresis, 72

Canary, 79

Cat model, 216

Cathodic current pulses, 216

CBA/CaJ mice, 233

Cell death, 79

Cell division, 79

Chick cochlear nucleus, 180

Childhood, 297

Cholesteatoma, 65

Cibacron Blue, 399

Cochlea, 362

Cochlear amplifier, 130

Cochlear degeneration, 118

Cochlear hair cell damage, 172

Cochlear hearing loss, 388

Cochlear implant, 87, 159, 216

Cochlear mechanics, 31

Cochlear nucleus angularis, 41

Cochlear phase response, 408
Cochlear trauma, 54

Combination-sensitive neurons, 104

Combination stimuli, 104

Contralateral effect, 268

Cortical development, 297

Cytoarchitectural maturation, 297

Dead regions, 172

Distortion product otoacoustic emission, 130, 233, 268

Distortion product otoacoustic emission adaptation, 31

Echidna, 130

Eighth nerve calyces, 180

Electric stimulation, 216

Electrode configuration, 87

Elevation localization, 246

Emission audiograms, 130

Envelope modulation, 159

Evoked potential, 216

Evolution, 130

Extracellular signal regulated kinases (ERK), 377

Feedback system, 31

Fetal period, 297

Fibrocytes, 118

Forward-masking paradigm, 216

Frequency map, 41

Frequency selectivity, 199

Frequency separation, 388

Frequency tuning, 199, 279

GABAergic drugs, 54

GABAergic inhibition, 279

GABAergic perisomatic puncta, 279

Gabapentin, 54

Gammachirp filter, 408

Gap duration discrimination, 388

Gap marker duration, 388

Gentamicin, 147

Gerbil auditory cortex, 279

Gerbil cochlea, 257

Gerbil posterior crista, 147

Group I mGluR-mediated calcium homeostasis, 180

Guinea pig, 199, 268, 362

Hair cell, 79, 172

Hair cell loss, 118

Harmonic complexes, 408

Head impulse test, 72

Head-related transfer function, 1

Head-turning behavior, 1

Hearing development, 312, 330, 348
Hearing loss, 118, 233, 388

Histology, 297

Histopathology, 118

Human thresholds, 189

Hypothyroidism, 312, 330, 348

hyt mice, 312, 330, 348

Ideal observer model, 189

Immunocytochemistry, 279

Immunohistochemistry, 297

Inbred mice, 233

Infancy, 297

Inferior colliculus, 104

Inflammation, 65

Informational masking, 189

Inner hair cell, 362

Inner hair cell function, 172

Inner hair cell loss, 118

Interaural time difference, 1

Intracellular $\mathrm{Ca}^{2+}, 362,399$

Intracellular transduction pathway, 377

Ipsilateral effect, 268

Keratin, 65

Laminar analysis, 279

Laminar cytoarchitecture, 297

Lateral lemniscus, 104

Local field potentials, 199

Longitudinal coupling, 257

Masker-probe interval, 216

Masking, 408

Maternal thyroid hormones, 330

Medial olivocochlear reflex, 31

Membrane capacitance, 362

Membrane conductance, 362

Metabotropic glutamate receptor (mGluR), 180

Mitochondrial DNA mutation, 172

Mitogen-activated protein kinases (MEK), 377

Modulation sensitivity, 159

Monotremes, 130

Mouse model, 65, 233

Mustached bat, 104

Neurite growth, 377

Neurofilaments, 297

Neuronal loss, 118

Neurotrophin-3, 377

Nitric oxide, 399

Noise, 159, 233

Noise exposure, 54

Nucleus angularis, 41 
Nucleus magnocellularis, 180

Nystagmus, 72

Off-vertical axis rotation, 22

Olivocochlear feedback, 268

Olivocochlear reflex, 31

Organ of Corti, 257

Osteoclasts, 65

Osteolysis, 65

Otolith organ, 22

Ototoxic drugs, 147

Outer hair cell function, 172

Outer hair cell loss, 118

P2X receptor, 362

p38, 377

Peripheral auditory system, 130

Phase, 408

Posterior crista, 147

Potassium ion currents, 362

Presbycusis, 118

Prosthetic noise, 159

Prosthetic sensory system, 159

Protein kinase G, 399

Psychophysics, 1, 54, 172, 189, 246, 408

Purinergic receptor, 399

Pyridoxal phosphate 6-azophenyl-2', $4^{\prime}$ disulfonic acid, 399
Ras, 377

Rat model, 54, 377

Refractory period, 216

Search coil, 72

Semicircular canal, 22

Sensory cells, 147

Sound intensity, 41

Sound localization, 1, 246

Sound overexposure, 233

Spatial hearing, 1

SPEAK processing strategy, 87

Spectral integration, 104

Speech perception, 87

Spetum cruciatum, 147

Spiral ganglion neurons, 377

Spiral ligament, 118

Spiral limbus, 118

Stimulation placement, 87

Stimulus intensity, 246

Stochastic resonance, 159

Streptomycin, 147

Stria vascularis, 118

Supporting cells, 147, 399

Suramin, 399

Tachyglossus aculeatus, 130
Thyroid hormone, 312, 330, 348

Thyroid stimulating hormone, 312 , 330, 348

Thyrotropin, 348

Thyrotropin receptor, 312

Thyroxin, 312, 330, 348

Tinnitus, 54

Tonotopic organization, 41, 362

Tshr gene, 312

Tshr mutant (hyt) mice, 312, 330, 348

TUNEL, 79

2-deoxyglucose (2-DG) uptake, 312

Uncertainty, 189

Velocity storage, 22

Vestibular end organs, 147

Vestibular hypofunction, 72

Vestibular neuritis, 72

Vestibulo-ocular reflex, 22, 72

Virtual space, 1

WB/ReJ mice, 233

Yound adulthood, 297

Zinc, 180 